ESAIM: M2AN 49 (2015) 373-394

DOI: $10.1051 / \mathrm{m} 2 \mathrm{an} / 2014037$
ESAIM: Mathematical Modelling and Numerical Analysis

www.esaim-m2an.org

\title{
DISCRETIZED FRACTIONAL SUBSTANTIAL CALCULUS *
}

\author{
Minghua Chen $^{1}$ and Weihua Deng ${ }^{1}$
}

\begin{abstract}
This paper discusses the properties and the numerical discretizations of the fractional substantial integral

$$
I_{s}^{\nu} f(x)=\frac{1}{\Gamma(\nu)} \int_{a}^{x}(x-\tau)^{\nu-1} \mathrm{e}^{-\sigma(x-\tau)} f(\tau) \mathrm{d} \tau, \quad \nu>0
$$

and the fractional substantial derivative

$$
D_{s}^{\mu} f(x)=D_{s}^{m}\left[I_{s}^{\nu} f(x)\right], \quad \nu=m-\mu
$$

where $D_{s}=\frac{\partial}{\partial x}+\sigma=D+\sigma, \sigma$ can be a constant or a function not related to $x$, say $\sigma(y)$; and $m$ is the smallest integer that exceeds $\mu$. The Fourier transform method and fractional linear multistep method are used to analyze the properties or derive the discretized schemes. And the convergences of the presented discretized schemes with the global truncation error $\mathcal{O}\left(h^{p}\right)(p=1,2,3,4,5)$ are theoretically proved and numerically verified.
\end{abstract}

Mathematics Subject Classification. 26A33, 65L06, 42A38, 65M12.

Received January 5, 2014. Revised August 15, 2014

Published online February 19, 2015.

\section{INTRODUCTION}

Anomalous diffusion processes are usually characterized by the nonlinear time dependance of the mean squared displacement, i.e., $\left\langle z^{2}(t)\right\rangle \sim t^{\alpha}[1]$. When $0<\alpha<1$, it is called subdiffusion; $\alpha>1$ corresponds to superdiffusion, and $\alpha=1$ to normal diffusion. A versatile framework for describing the anomalous diffusion is the continuous time random walks (CTRWs), which is governed by the waiting time probability density function (PDF) and jump length PDF. When the waiting time PDF and/or jump length PDF are power-law, and the two PDFs are independent, the transport equations can be derived, namely fractional Fokker-Planck and KleinKramers equations [12]. Usually the time fractional Fokker-Planck equation characterizes the subdiffusion, whereas the space fractional Fokker-Planck equation depicts the Lévy flight [13]. The Lévy flight has a diverging mean squared displacement, and can just be applied to rather exotic physical processes [18].

Keywords and phrases. Fractional substantial calculus, fractional linear multistep methods, fourier transform, stability and convergence.

* This work was supported by the National Natural Science Foundation of China under Grant No. 11271173.

1 School of Mathematics and Statistics, Gansu Key Laboratory of Applied Mathematics and Complex Systems, Lanzhou

University, Lanzhou 730000, P.R. China. chenmh2009@lzu.edu.cn; dengwh@lzu.edu.cn 
Lévy walk [14] gives another proper dynamical description for the superdiffusion (roughly speaking, now the particle has finite physical speed), and the PDFs of waiting time and jump length are spatiotemporal coupling [18]. Friedrich and his co-workers discuss the CTRW model with position-velocity coupling PDF [8]. Carmi and Barkai use the CTRW model with functional of path and position coupling PDF [2]. Based on the CTRW models with coupling PDFs, they also derive the deterministic equations; and mathematically an important operator, fractional substantial derivative, is introduced $[2,3,8,18,20]$.

With the wide applications of the fractional substantial derivative, it seems to be urgent to mathematically analyze its properties and numerically provide its effective discretizations. This paper focuses on these two topics. The fractional substantial derivative, firstly introduced in [8] and further applied in [2], is defined by

$$
D_{s}^{1-\nu} f(x)=\frac{1}{\Gamma(\nu)}\left[\frac{\partial}{\partial x}+\sigma\right] \int_{0}^{x}(x-\tau)^{\nu-1} \mathrm{e}^{-\sigma(x-\tau)} f(\tau) d \tau, \quad 0<\nu<1,
$$

where $\sigma$ can be a constant or a function not related to $x$, say, $\sigma(y)$. In this paper, we extend the order of fractional substantial derivative $\nu \in(0,1)$ to $\nu>0$. First, we introduce the fractional substantial integral.

Definition 1.1. Let $\nu>0, f(x)$ be piecewise continuous on $(a, \infty)$ and integrable on any finite subinterval $[a, \infty)$; and let $\sigma$ be a constant or a function without related to $x$. Then the fractional substantial integral of $f$ of order $\nu$ is defined as

$$
I_{s}^{\nu} f(x)=\frac{1}{\Gamma(\nu)} \int_{a}^{x}(x-\tau)^{\nu-1} \mathrm{e}^{-\sigma(x-\tau)} f(\tau) d \tau, \quad x>a .
$$

Definition 1.2. Let $\mu>0, f(x)$ be $(m-1)$-times continuously differentiable on $(a, \infty)$ and its m-times derivative be integrable on any finite subinterval of $[a, \infty)$, where $m$ is the smallest integer that exceeds $\mu$; and let $\sigma$ be a constant or a function without related to $x$. Then the fractional substantial derivative of $f$ of order $\mu$ is defined as

$$
D_{s}^{\mu} f(x)=D_{s}^{m}\left[I_{s}^{m-\mu} f(x)\right]
$$

where

$$
D_{s}^{m}=\left(\frac{\partial}{\partial x}+\sigma\right)^{m}=(D+\sigma)^{m}=(D+\sigma)(D+\sigma) \ldots(D+\sigma)
$$

When $\sigma=0$, obviously, the fractional substantial integral and derivative reduce to the Riemann-Liouville fractional integral and derivative, respectively.

In the following, using Fourier transform methods and fractional linear multistep methods, respectively, we derive the $p$ th order $(p \leq 5)$ approximations of the $\alpha$ th fractional substantial derivative $(\alpha>0)$ or fractional substantial integral $(\alpha<0)$ by the corresponding coefficients of the generating functions $\kappa^{p, \alpha}(\zeta)$, with

$$
\kappa^{p, \alpha}(\zeta)=\left(\sum_{i=1}^{p} \frac{1}{i}\left(1-\mathrm{e}^{-\sigma h} \zeta\right)^{i}\right)^{\alpha},
$$

where $h$ is the uniform stepsize. We can rewrite (1.4) as a tabular; see Table 1.

For $\sigma=0$, formula (1.4) reduces to the fractional Lubich's methods [11]. For $\sigma=0$ and $\alpha=1$, the scheme reduces to the classical $(p+1)$-point backward difference formula [10].

The outline of this paper is as follows. In Section 2, we give some properties of the fractional substantial calculus. In Sections 3 and 4, using Fourier transform method and fractional linear multistep method, respectively, we derive the convergence of the discretized schemes of the fractional substantial calculus. And the convergence with the global truncation error $\mathcal{O}\left(h^{p}\right)(p=1,2,3,4,5)$ are numerically verified in Section 5 . Finally, we conclude the paper with some remarks in the last section. 
TABLE 1. Generating functions of the coefficients of (3.5) or (1.4) for the $p$ th order approximation of $\alpha$ th fractional substantial derivative.

\begin{tabular}{ll}
\hline$p$ & \multicolumn{1}{c}{$\kappa^{p, \alpha}(\zeta)$} \\
\hline 1 & $\left(1-\mathrm{e}^{-\sigma h} \zeta\right)^{\alpha}$ \\
2 & $\left(3 / 2-2 \mathrm{e}^{-\sigma h} \zeta+1 / 2\left(\mathrm{e}^{-\sigma h} \zeta\right)^{2}\right)^{\alpha}$ \\
3 & $\left(11 / 6-3 \mathrm{e}^{-\sigma h} \zeta+3 / 2\left(\mathrm{e}^{-\sigma h} \zeta\right)^{2}-1 / 3\left(\mathrm{e}^{-\sigma h} \zeta\right)^{3}\right)^{\alpha}$ \\
4 & $\left(25 / 12-4 \mathrm{e}^{-\sigma h} \zeta+3\left(\mathrm{e}^{-\sigma h} \zeta\right)^{2}-4 / 3\left(\mathrm{e}^{-\sigma h} \zeta\right)^{3}+1 / 4\left(\mathrm{e}^{-\sigma h} \zeta\right)^{4}\right)^{\alpha}$ \\
5 & $\left(137 / 60-5 \mathrm{e}^{-\sigma h} \zeta+5\left(\mathrm{e}^{-\sigma h} \zeta\right)^{2}-10 / 3\left(\mathrm{e}^{-\sigma h} \zeta\right)^{3}+5 / 4\left(\mathrm{e}^{-\sigma h} \zeta\right)^{4}-1 / 5\left(\mathrm{e}^{-\sigma h} \zeta\right)^{5}\right)^{\alpha}$ \\
\hline
\end{tabular}

\section{Properties for the fractional substantial CAlCulus}

Let us now consider some properties of the fractional substantial calculus.

Lemma 2.1. Let $f(x)$ be continuous on $[a, \infty)$, and $\nu>0$. Then for all $x \geq a$,

$$
\lim _{\nu \rightarrow 0} I_{s}^{\nu} f(x)=f(x) .
$$

Hence we can put $I_{s}^{0} f(x)=f(x)$.

Proof. If $f(x)$ has continuous derivative for $x \geq a$, then using integration by parts to (1.1), there exists

$$
\begin{aligned}
I_{s}^{\nu} f(x) & =-\frac{1}{\Gamma(\nu+1)} \int_{a}^{x} \mathrm{e}^{-\sigma(x-\tau)} f(\tau) \mathrm{d}(x-\tau)^{\nu} \\
& =\frac{(x-a)^{\nu} \mathrm{e}^{-\sigma(x-a)} f(a)}{\Gamma(\nu+1)}+\frac{1}{\Gamma(\nu+1)} \int_{a}^{x}(x-\tau)^{\nu} \mathrm{e}^{-\sigma(x-\tau)} D_{s} f(\tau) \mathrm{d} \tau,
\end{aligned}
$$

where $D_{s}$ is defined by (1.3). So we get

$$
\lim _{\nu \rightarrow 0} I_{s}^{\nu} f(x)=\mathrm{e}^{-\sigma(x-a)} f(a)+\sigma \int_{a}^{x} \mathrm{e}^{-\sigma(x-\tau)} f(\tau) \mathrm{d} \tau+\int_{a}^{x} \mathrm{e}^{-\sigma(x-\tau)} \mathrm{d} f(\tau)=f(x) .
$$

If $f(x)$ is only continuous for $x \geq a$, the similar arguments can be performed as ([17], pp. 66 and 67 ), we omit it here.

Lemma 2.2. Let $f(x)$ be continuous on $[a, \infty)$ and $\mu, \nu>0$. Then for all $x \geq a$,

$$
I_{s}^{\nu}\left[I_{s}^{\mu} f(x)\right]=I_{s}^{\mu+\nu} f(x)=I_{s}^{\mu}\left[I_{s}^{\nu} f(x)\right] .
$$

Proof.

$$
\begin{aligned}
I_{s}^{\nu}\left[I_{s}^{\mu} f(x)\right] & =\frac{1}{\Gamma(\nu)} \int_{a}^{x}(x-\tau)^{\nu-1} \mathrm{e}^{-\sigma(x-\tau)}\left[I_{s}^{\mu} f(\tau)\right] \mathrm{d} \tau \\
& =\frac{1}{\Gamma(\mu) \Gamma(\nu)} \int_{a}^{x}(x-\tau)^{\nu-1} \mathrm{e}^{-\sigma(x-\tau)} \mathrm{d} \tau \int_{a}^{\tau}(\tau-\xi)^{\mu-1} \mathrm{e}^{-\sigma(\tau-\xi)} f(\xi) \mathrm{d} \xi \\
& =\frac{1}{\Gamma(\mu) \Gamma(\nu)} \int_{a}^{x} \mathrm{e}^{-\sigma(x-\xi)} f(\xi) \mathrm{d} \xi \int_{\xi}^{x}(x-\tau)^{\nu-1}(\tau-\xi)^{\mu-1} d \tau \\
& =I_{s}^{\mu+\nu} f(x)
\end{aligned}
$$

where the integral

$$
\int_{\xi}^{x}(x-\tau)^{\nu-1}(\tau-\xi)^{\mu-1} \mathrm{~d} \tau=\frac{\Gamma(\mu) \Gamma(\nu)}{\Gamma(\mu+\nu)}(x-\xi)^{\mu+\nu-1} .
$$


Lemma 2.3. Let $f(x)$ be (m-1)-times continuously differentiable on $(a, \infty)$ and its $m$-times derivative be integrable on any finite subinterval of $[a, \infty)$ and $\nu>0$, where $m$ is the smallest integer that exceeds $\nu$. Then for all $x \geq a$,

$$
D_{s}^{\nu}\left[I_{s}^{\nu} f(x)\right]=f(x) .
$$

Proof. Let us first consider the case of integer $\nu=m \geq 1$ :

$$
\begin{aligned}
D_{s}^{m}\left[I_{s}^{m} f(x)\right] & =D_{s}^{m}\left[\frac{1}{(m-1) !} \int_{a}^{x}(x-\tau)^{m-1} \mathrm{e}^{-\sigma(x-\tau)} f(\tau) \mathrm{d} \tau\right] \\
& =D_{s} \int_{a}^{x} \mathrm{e}^{-\sigma(x-\tau)} f(\tau) d \tau=D_{s}\left[I_{s} f(x)\right]=f(x) .
\end{aligned}
$$

For $m-1<\nu<m$, from Lemma 2.2, there exists

$$
I_{s}^{m}=I_{s}^{m-\nu}\left[I_{s}^{\nu} f(x)\right] .
$$

Thus, using (1.2) and above equation, we obtain

$$
D_{s}^{\nu}\left[I_{s}^{\nu} f(x)\right]=D_{s}^{m}\left\{I_{s}^{m-\nu}\left[I_{s}^{\nu} f(x)\right]\right\}=D_{s}^{m}\left[I_{s}^{m} f(x)\right]=f(x) .
$$

Lemma 2.4. Let $f(x)$ be $(r-1)$-times continuously differentiable on $(a, \infty)$ and its $r$-times derivative be integrable on any finite subinterval of $[a, \infty)$, where $r=\max (m, n), m$ and $n$ are positive integers. Denoting that

$$
m-\nu=n-\mu, \quad \mu>0, \nu>0,
$$

then for all $x \geq a$,

$$
D_{s}^{n}\left[I_{s}^{\mu} f(x)\right]=D_{s}^{m}\left[I_{s}^{\nu} f(x)\right]
$$

Proof. If $m=n$, the lemma is trivial. Supposing that $n>m$ and $\gamma=n-m>0$, it yields $\mu=\nu+\gamma>0$. Then according to Lemmas 2.2 and 2.3, we obtain

$$
D_{s}^{\gamma}\left[I_{s}^{\nu+\gamma} f(x)\right]=D_{s}^{\gamma}\left[I_{s}^{\gamma} I_{s}^{\nu} f(x)\right]=I_{s}^{\nu} f(x) .
$$

Letting $D_{s}^{m}$ perform on both sides of the above equation leads to

$$
D_{s}^{m+\gamma}\left[I_{s}^{\nu+\gamma} f(x)\right]=D_{s}^{m}\left[I_{s}^{\nu} f(x)\right]
$$

that is

$$
D_{s}^{n}\left[I_{s}^{\mu} f(x)\right]=D_{s}^{m}\left[I_{s}^{\nu} f(x)\right] .
$$

Lemma 2.5. Let $f(x)$ be continuously differentiable on $[a, \infty)$, and $\nu>0$. Then for all $x \geq a$,

$$
I_{s}^{\nu+1}\left[D_{s} f(x)\right]=I_{s}^{\nu} f(x)-\frac{f(a)}{\Gamma(\nu+1)}(x-a)^{\nu} \mathrm{e}^{-\sigma(x-a)} ;
$$

and

$$
D_{s}\left[I_{s}^{\nu} f(x)\right]=I_{s}^{\nu}\left[D_{s} f(x)\right]+\frac{f(a)}{\Gamma(\nu)}(x-a)^{\nu-1} \mathrm{e}^{-\sigma(x-a)} .
$$


Proof. Using integration by parts, it is easy to get

$$
I_{s}^{\nu} f(x)=\frac{f(a)}{\Gamma(\nu+1)}(x-a)^{\nu} \mathrm{e}^{-\sigma(x-a)}+\frac{1}{\Gamma(\nu+1)} \int_{a}^{x}(x-\tau)^{\nu} \mathrm{e}^{-\sigma(x-\tau)}\left[D_{s} f(\tau)\right] \mathrm{d} \tau,
$$

where $D_{s}$ is defined by (1.3). Thus we obtain (2.1).

Next we prove (2.2). From (2.1), it leads to

$$
\begin{aligned}
D_{s}\left[I_{s}^{\nu} f(x)\right] & =D_{s}\left\{I_{s}^{\nu+1}\left[D_{s} f(x)\right]+\frac{f(a)}{\Gamma(\nu+1)}(x-a)^{\nu} \mathrm{e}^{-\sigma(x-a)}\right\} \\
& =I_{s}^{\nu}\left[D_{s} f(x)\right]+\frac{f(a)}{\Gamma(\nu+1)}(D+\sigma)\left[(x-a)^{\nu} \mathrm{e}^{-\sigma(x-a)}\right] \\
& =I_{s}^{\nu}\left[D_{s} f(x)\right]+\frac{f(a)}{\Gamma(\nu)}(x-a)^{\nu-1} \mathrm{e}^{-\sigma(x-a)} .
\end{aligned}
$$

Hence, we get (2.2).

Lemma 2.6. Let $f(x)$ be (m-1)-times continuously differentiable on $(a, \infty)$ and its $m$-times derivative be integrable on any finite subinterval of $[a, \infty), \mu>0, \nu>0$; and $m$ is the smallest integer that exceeds $\mu$. Then for all $x \geq a$,

$$
I_{s}^{\nu} f(x)=I_{s}^{m+\nu}\left[D_{s}^{m} f(x)\right]+\sum_{k=0}^{m-1} \frac{D_{s}^{k} f(a)(x-a)^{k+\nu} \mathrm{e}^{-\sigma(x-a)}}{\Gamma(k+\nu+1)}
$$

and

$$
\begin{aligned}
D_{s}^{\mu} f(x) & =I_{s}^{m-\mu}\left[D_{s}^{m} f(x)\right]+\sum_{k=0}^{m-1} \frac{D_{s}^{k} f(a)(x-a)^{k-\mu} \mathrm{e}^{-\sigma(x-a)}}{\Gamma(k-\mu+1)} \\
& ={ }^{C} D_{s}^{\mu} f(x)+\sum_{k=0}^{m-1} \frac{D_{s}^{k} f(a)(x-a)^{k-\mu} \mathrm{e}^{-\sigma(x-a)}}{\Gamma(k-\mu+1)}
\end{aligned}
$$

where ${ }^{C} D_{s}^{\mu} f(x)=I_{s}^{m-\mu}\left[D_{s}^{m} f(x)\right]$ can be similarly called Caputo fractional substantial derivative [17]. In particular, from (2.3) and (2.4), we can extend the definitions of $I_{s}^{\nu}$ and $D_{s}^{\mu}$, i.e., $\mu, \nu$ can belong to $\mathbb{R}$ instead of being limited to $\mathbb{R}^{+}$, then for any real $\alpha$, there exists

$$
I_{s}^{\alpha}=D_{s}^{-\alpha},
$$

i.e., if taking $\nu=\alpha$ and $\mu=-\alpha$ in (2.3) and (2.4), $I_{s}^{\alpha} f(x)=D_{s}^{-\alpha} f(x)$.

Proof. Replacing $\nu$ by $\nu+1$ and $f$ by $D_{s} f$ in (2.1), we obtain

$$
I_{s}^{\nu+1}\left[D_{s} f(x)\right]=I_{s}^{\nu+2}\left[D_{s}^{2} f(x)\right]+\frac{D_{s} f(a)}{\Gamma(\nu+2)}(x-a)^{\nu+1} \mathrm{e}^{-\sigma(x-a)} .
$$

Thus, according to the above equation and (2.1), there exists

$$
\begin{aligned}
I_{s}^{\nu} f(x) & =I_{s}^{\nu+1}\left[D_{s} f(x)\right]+\frac{f(a)}{\Gamma(\nu+1)}(x-a)^{\nu} \mathrm{e}^{-\sigma(x-a)} \\
& =I_{s}^{\nu+2}\left[D_{s}^{2} f(x)\right]+\frac{D_{s} f(a)}{\Gamma(\nu+2)}(x-a)^{\nu+1} \mathrm{e}^{-\sigma(x-a)}+\frac{f(a)}{\Gamma(\nu+1)}(x-a)^{\nu} \mathrm{e}^{-\sigma(x-a)} \\
& =I_{s}^{(\nu+m)}\left[D_{s}^{m} f(x)\right]+\sum_{k=0}^{m-1} \frac{D_{s}^{k} f(a)(x-a)^{\nu+k} \mathrm{e}^{-\sigma(x-a)}}{\Gamma(\nu+k+1)} .
\end{aligned}
$$


To prove (2.4), letting $D_{s}$ perform on both sides of (2.2) leads to

$$
D_{s}^{2}\left[I_{s}^{\nu} f(x)\right]=D_{s}\left\{I_{s}^{\nu}\left[D_{s} f(x)\right]\right\}+\frac{f(a)}{\Gamma(\nu-1)}(x-a)^{\nu-2} \mathrm{e}^{-\sigma(x-a)},
$$

and replacing $f$ with $D_{s} f$ in $(2.2)$ yields

$$
D_{s}\left\{I_{s}^{\nu}\left[D_{s} f(x)\right]\right\}=I_{s}^{\nu}\left[D_{s}^{2} f(x)\right]+\frac{D_{s} f(a)}{\Gamma(\nu)}(x-a)^{\nu-1} \mathrm{e}^{-\sigma(x-a)} .
$$

Therefore, there exists

$$
D_{s}^{2}\left[I_{s}^{\nu} f(x)\right]=I_{s}^{\nu}\left[D_{s}^{2} f(x)\right]+\frac{D_{s} f(a)}{\Gamma(\nu)}(x-a)^{\nu-1} \mathrm{e}^{-\sigma(x-a)}+\frac{f(a)}{\Gamma(\nu-1)}(x-a)^{\nu-2} \mathrm{e}^{-\sigma(x-a)} .
$$

Repeating the procedure $m-1$ times results in

$$
D_{s}^{m}\left[I_{s}^{\nu} f(x)\right]=I_{s}^{\nu}\left[D_{s}^{m} f(x)\right]+\sum_{k=0}^{m-1} \frac{D_{s}^{k} f(a)(x-a)^{\nu+k-m} \mathrm{e}^{-\sigma(x-a)}}{\Gamma(\nu+k-m+1)} .
$$

Taking $\nu=m-\mu$, then equation (2.6) can be rewritten as

$$
D_{s}^{\mu} f(x)=D_{s}^{m}\left[I_{s}^{\nu} f(x)\right]=I_{s}^{m-\mu}\left[D_{s}^{m} f(x)\right]+\sum_{k=0}^{m-1} \frac{D_{s}^{k} f(a)(x-a)^{k-\mu} \mathrm{e}^{-\sigma(x-a)}}{\Gamma(k-\mu+1)} .
$$

From (2.3) and (2.4), it yields that $I_{s}^{\alpha}=D_{s}^{-\alpha}$ for any real $\alpha$.

Lemma 2.7. Let $f(x)$ be $(m-1)$-times continuously differentiable on $(a, \infty)$ and its $m$-times derivative be integrable on any finite subinterval of $[a, \infty)$ and $\nu>0$, where $m$ is the smallest integer that exceeds $\nu$. Then for all $x>a$,

$$
I_{s}^{\nu}\left[D_{s}^{\nu} f(x)\right]=f(x)-\sum_{j=1}^{m}\left[D_{s}^{\nu-j} f(x)\right]_{x=a} \frac{(x-a)^{\nu-j} \mathrm{e}^{-\sigma(x-a)}}{\Gamma(\nu-j+1)}
$$

Proof. On the one hand, there exists

$$
I_{s}^{\nu}\left[D_{s}^{\nu} f(x)\right]=D_{s}\left\{\frac{1}{\Gamma(\nu+1)} \int_{a}^{x}(x-\tau)^{\nu} \mathrm{e}^{-\sigma(x-\tau)}\left[D_{s}^{\nu} f(\tau)\right] \mathrm{d} \tau\right\}
$$


On the other hand, repeatedly integrating by parts and using Lemma 2.2 we have

$$
\begin{aligned}
& \frac{1}{\Gamma(\nu+1)} \int_{a}^{x}(x-\tau)^{\nu} \mathrm{e}^{-\sigma(x-\tau)} D_{s}^{\nu} f(\tau) \mathrm{d} \tau \\
&=\frac{1}{\Gamma(\nu+1)} \int_{a}^{x}(x-\tau)^{\nu} \mathrm{e}^{-\sigma(x-\tau)} D_{s}^{m}\left[I_{s}^{m-\nu} f(\tau)\right] \mathrm{d} \tau \\
&=\frac{1}{\Gamma(\nu)} \int_{a}^{x}(x-\tau)^{\nu-1} \mathrm{e}^{-\sigma(x-\tau)} D_{s}^{m-1}\left[I_{s}^{m-\nu} f(\tau)\right] \mathrm{d} \tau \\
&-\frac{(x-a)^{\nu} \mathrm{e}^{-\sigma(x-a)}}{\Gamma(\nu+1)}\left\{D_{s}^{m-1}\left[I_{s}^{m-\nu} f(x)\right]\right\}_{x=a} \\
&= \frac{1}{\Gamma(\nu-m+1)} \int_{a}^{x}(x-\tau)^{\nu-m} \mathrm{e}^{-\sigma(x-\tau)}\left[I_{s}^{m-\nu} f(\tau)\right] \mathrm{d} \tau \\
&-\sum_{j=1}^{m}\left\{D_{s}^{m-j}\left[I_{s}^{m-\nu} f(x)\right]\right\}_{x=a} \frac{(x-a)^{\nu-j+1} \mathrm{e}^{-\sigma(x-a)}}{\Gamma(\nu-j+2)} \\
&= I_{s}^{\nu-m+1}\left[I_{s}^{m-\nu} f(\tau)\right]-\sum_{j=1}^{m}\left[D_{s}^{\nu-j} f(x)\right]_{x=a} \frac{(x-a)^{\nu-j+1} \mathrm{e}^{-\sigma(x-a)}}{\Gamma(\nu-j+2)} \\
&= I_{s} f(\tau)-\sum_{j=1}^{m}\left[D_{s}^{\nu-j} f(x)\right]_{x=a} \frac{(x-a)^{\nu-j+1} \mathrm{e}^{-\sigma(x-a)}}{\Gamma(\nu-j+2)} .
\end{aligned}
$$

Combining (2.7) and (2.8), we obtain

$$
I_{s}^{\nu}\left[D_{s}^{\nu} f(x)\right]=f(x)-\sum_{j=1}^{m}\left[D_{s}^{\nu-j} f(x)\right]_{x=a} \frac{(x-a)^{\nu-j} \mathrm{e}^{-\sigma(x-a)}}{\Gamma(\nu-j+1)} .
$$

Lemma 2.8. Let $f(x)$ be (m-1)-times continuously differentiable on $(a, \infty)$ and its $m$-times derivative be integrable on any finite subinterval of $[a, \infty)$ and $\mu>0, \nu>0$, where $m$ is the smallest integer that exceeds $\mu$. Then for all $x>a$,

$$
D_{s}^{\mu}\left[D_{s}^{-\nu} f(x)\right]=D_{s}^{\mu-\nu} f(x) .
$$

Proof. Two cases must be considered: $\mu>\nu \geq 0$ and $\nu \geq \mu \geq 0$.

Case $\mu>\nu \geq 0$ : taking $0 \leq n-1 \leq \mu-\nu<n, n$ is an integer and using $0 \leq m-1 \leq \mu<m$, then from (2.5) and (1.2) and Lemmas 2.2 and 2.4, we have

$$
\begin{aligned}
D_{s}^{\mu}\left[D_{s}^{-\nu} f(x)\right] & =D_{s}^{\mu}\left[I_{s}^{\nu} f(x)\right]=D_{s}^{m}\left\{I_{s}^{m-\mu}\left[I_{s}^{\nu} f(x)\right]\right\}=D_{s}^{m}\left\{I_{s}^{m-\mu+\nu} f(x)\right\} \\
& =D_{s}^{n}\left\{I_{s}^{n-\mu+\nu} f(x)\right\}=D_{s}^{\mu-\nu} f(x) .
\end{aligned}
$$

Case $\nu \geq \mu \geq 0$ : according to Lemmas 2.2 and 2.3 , we obtain

$$
D_{s}^{\mu}\left[I_{s}^{\nu} f(x)\right]=D_{s}^{\mu}\left[I_{s}^{\mu} I_{s}^{\nu-\mu} f(x)\right]=D_{s}^{\mu-\nu} f(x) .
$$

Lemma 2.9. Let $f(x)$ be $(m-1)$-times continuously differentiable on $(a, \infty)$ and its $m$-times derivative be integrable on any finite subinterval of $[a, \infty)$ and $\mu>0, \nu>0$, where $m$ is the smallest integer that exceeds $\nu$. Then for all $x>a$,

$$
D_{s}^{-\mu}\left[D_{s}^{\nu} f(x)\right]=D_{s}^{\nu-\mu} f(x)-\sum_{j=1}^{m}\left[D_{s}^{\nu-j} f(x)\right]_{x=a} \frac{(x-a)^{\mu-j} \mathrm{e}^{-\sigma(x-a)}}{\Gamma(\mu-j+1)} .
$$


Proof. If $\nu \leq \mu$, there exists $D_{s}^{-\mu}=D_{s}^{\nu-\mu} D_{s}^{-\nu}$ by Lemma 2.2; and if $\nu \geq \mu$, there also exists $D_{s}^{-\mu}=D_{s}^{\nu-\mu} D_{s}^{-\nu}$ by Lemma 2.8. Therefore, using Lemma 2.7 we have

$$
\begin{aligned}
D_{s}^{-\mu}\left[D_{s}^{\nu} f(x)\right] & =D_{s}^{\nu-\mu}\left\{D_{s}^{-\nu}\left[D_{s}^{\nu} f(x)\right]\right\} \\
& =D_{s}^{\nu-\mu}\left\{f(x)-\sum_{j=1}^{m}\left[D_{s}^{\nu-j} f(x)\right]_{x=a} \frac{(x-a)^{\nu-j} \mathrm{e}^{-\sigma(x-a)}}{\Gamma(\nu-j+1)}\right\} \\
& =D_{s}^{\nu-\mu} f(x)-\sum_{j=1}^{m}\left[D_{s}^{\nu-j} f(x)\right]_{x=a} \frac{(x-a)^{\mu-j} \mathrm{e}^{-\sigma(x-a)}}{\Gamma(\mu-j+1)},
\end{aligned}
$$

where we use the following formula

$$
D_{s}^{\mu}\left[\mathrm{e}^{-\sigma(x-a)}(x-a)^{\nu}\right]=\frac{\Gamma(\nu+1)}{\Gamma(\nu+1-\mu)}(x-a)^{\nu-\mu} \mathrm{e}^{-\sigma(x-a)},
$$

which can be similarly proven as the way in ([17], p. 56).

Lemma 2.10. Let $\mu>0, \nu>0$ and $f(x)$ be $(r-1)$-times continuously differentiable on $(a, \infty)$ and its $r$-times derivative be integrable on any finite subinterval of $[a, \infty)$, where $r=\max (m, n), m$ and $n$ is the smallest integer that exceeds $\mu$ and $\nu$, respectively. Then for all $x>a$,

$$
D_{s}^{\mu}\left[D_{s}^{\nu} f(x)\right]=D_{s}^{\mu+\nu} f(x)-\sum_{j=1}^{n}\left[D_{s}^{\nu-j} f(x)\right]_{x=a} \frac{(x-a)^{-\mu-j} \mathrm{e}^{-\sigma(x-a)}}{\Gamma(-\mu-j+1)} .
$$

Proof. Similar to the well-known property of integer-order derivatives:

$$
\frac{\mathrm{d}^{m}}{\mathrm{~d} x^{m}}\left(\frac{\mathrm{d}^{n} f(x)}{\mathrm{d} x^{n}}\right)=\frac{\mathrm{d}^{n}}{\mathrm{~d} x^{n}}\left(\frac{\mathrm{d}^{m} f(x)}{\mathrm{d} x^{m}}\right)=\frac{\mathrm{d}^{m+n} f(x)}{\mathrm{d} x^{m+n}},
$$

it is easy to check that

$$
D_{s}^{m}\left[D_{s}^{n} f(x)\right]=D_{s}^{n}\left[D_{s}^{m} f(x)\right]=D_{s}^{m+n} f(x) .
$$

Therefore, according to (1.2), the above equation, and Lemma 2.8, there exists

$$
D_{s}^{n}\left[D_{s}^{m-\alpha} f(x)\right]=D_{s}^{n+m}\left[I_{s}^{\alpha} f(x)\right]=D_{s}^{n+m-\alpha} f(x), \text { for } \alpha \in(0,1],
$$

and denoting that $\gamma=m-\alpha$, it leads to

$$
D_{s}^{n}\left[D_{s}^{\gamma} f(x)\right]=D_{s}^{n+\gamma} f(x) .
$$

According to (1.2), Lemma 2.9, and (2.10), we obtain

$$
\begin{aligned}
D_{s}^{\mu}\left[D_{s}^{\nu} f(x)\right] & =D_{s}^{m}\left\{D_{s}^{-(m-\mu)}\left[D_{s}^{\nu} f(x)\right]\right\} \\
& =D_{s}^{m}\left\{D_{s}^{\mu+\nu-m} f(x)-\sum_{j=1}^{n}\left[D_{s}^{\nu-j} f(x)\right]_{x=a} \frac{(x-a)^{m-\mu-j} \mathrm{e}^{-\sigma(x-a)}}{\Gamma(m-\mu-j+1)}\right\} \\
& =D_{s}^{\mu+\nu} f(x)-\sum_{j=1}^{n}\left[D_{s}^{\nu-j} f(x)\right]_{x=a} \frac{(x-a)^{-\mu-j} \mathrm{e}^{-\sigma(x-a)}}{\Gamma(-\mu-j+1)} .
\end{aligned}
$$

Similar to the proof of ([17], pp. 76 and 77), we have the following remarks. 
Remark 2.11. If $D_{s}^{\mu} f(x)$ exists and is integrable, then the fractional substantial derivative $D_{s}^{\nu} f(x)$ also exists and is integrable for $0<\nu<\mu$.

Remark 2.12. Let $f(x)$ be (m-1)-times continuously differentiable on $(a, \infty)$ and its $m$-times derivative be integrable on any finite subinterval of $[a, \infty)$. Then for all $x \geq a$,

$$
\left[D_{s}^{\mu} f(x)\right]_{x=a}=0, \quad m-1 \leq \mu<m,
$$

if and only if

$$
D_{s}^{(j)} f(a)=0, \text { for } j=0,1, \ldots, m-1 .
$$

\section{Discretizations of Fractional substantial CAlCulus and its COnvergence; FOURIER TRANSFORM METHODS}

In this section, we derive the discretization schemes of fractional substantial calculus and prove their convergence by Fourier transform method.

Lemma 3.1. Let $\nu>0, f(x) \in L^{q}(\mathbb{R}), q \geq 1$, and

$$
\mathcal{I}_{s}^{\nu} f(x)=\frac{1}{\Gamma(\nu)} \int_{-\infty}^{x}(x-\tau)^{\nu-1} \mathrm{e}^{-\sigma(x-\tau)} f(\tau) \mathrm{d} \tau .
$$

Then

$$
\mathcal{F}\left(\mathcal{I}_{s}^{\nu} f(x)\right)=(\sigma-i \omega)^{-\nu} \widehat{f}(\omega)
$$

where $\mathcal{F}$ denotes Fourier transform operator and $\widehat{f}(\omega)=\mathcal{F}(f)$, i.e.,

$$
\widehat{f}(\omega)=\int_{\mathbb{R}} \mathrm{e}^{i \omega x} f(x) \mathrm{d} x .
$$

Proof. Taking the fractional substantial integral (1.1) with the lower terminal $a=-\infty$, equation (1.1) reduces to $(3.1)$.

Let us start with the Laplace transform of the function

$$
h(x)=\frac{x^{\nu-1}}{\Gamma(\nu)} \mathrm{e}^{-\sigma x}
$$

i.e.,

$$
\frac{1}{\Gamma(\nu)} \int_{0}^{\infty} x^{\nu-1} \mathrm{e}^{-(\sigma+s) x} d x=(\sigma+s)^{-\nu}
$$

where we use the well-known Laplace transform of the function $x^{\nu-1}$

$$
L\left\{x^{\nu-1} ; s\right\}=\int_{0}^{\infty} x^{\nu-1} \mathrm{e}^{-s x} d x=\Gamma(\nu) s^{-\nu} .
$$

It follows from the Dirichlet theorem ([7], p. 564) that the integral (3.2) converges if $\nu>0$. Taking $s=-i \omega$, where $\omega$ is real, we immediately have the Fourier transform of the function

$$
h_{+}(x)= \begin{cases}\frac{x^{\nu-1}}{\Gamma(\nu)} \mathrm{e}^{-\sigma x}, & x>0 \\ 0, & x \leq 0\end{cases}
$$


in the form

$$
\mathcal{F}\left(h_{+}(x)\right)=\int_{-\infty}^{\infty} h_{+}(x) \mathrm{e}^{i \omega x} d x=\frac{1}{\Gamma(\nu)} \int_{0}^{\infty} x^{\nu-1} \mathrm{e}^{-(\sigma-i \omega) x} d x=(\sigma-i \omega)^{-\nu}
$$

Since

$$
\mathcal{I}_{s}^{\nu} f(x)=\frac{1}{\Gamma(\nu)} \int_{-\infty}^{x}(x-\tau)^{\nu-1} \mathrm{e}^{-\sigma(x-\tau)} f(\tau) d \tau=\frac{x^{\nu-1} \mathrm{e}^{-\sigma x}}{\Gamma(\nu)} * f(x)=h(x) * f(x),
$$

where the asterisk means the convolution, then we have

$$
\mathcal{F}\left(\mathcal{I}_{s}^{\nu} f(x)\right)=\mathcal{F}(h(x) * f(x))=\mathcal{F}(h(x)) \cdot \mathcal{F}(f(x))=(\sigma-i \omega)^{-\nu} \widehat{f}(\omega) .
$$

Lemma 3.2. Let $\nu>0, f \in C_{0}^{m-1}(a, \infty)$ and its m-times derivative be integrable on any finite subinterval of $[a, \infty)$. Denoting that

$$
\mathcal{D}_{s}^{\nu} f(x)=D_{s}^{m}\left[\mathcal{I}_{s}^{m-\nu} f(x)\right]
$$

where $m$ is the smallest integer that exceeds $\nu$ and $D_{s}^{m}$ and $\mathcal{I}_{s}^{m-\nu}$ are defined by (1.3) and (3.1), respectively. Then

$$
\mathcal{F}\left(\mathcal{D}_{s}^{\nu} f(x)\right)=(\sigma-i \omega)^{\nu} \widehat{f}(\omega) .
$$

Proof. Taking the lower terminal $a=-\infty$ and using (2.4), we obtain

$$
\mathcal{D}_{s}^{\nu} f(x)=D_{s}^{m}\left[\mathcal{I}_{s}^{m-\nu} f(x)\right]=\mathcal{I}_{s}^{m-\nu}\left[D_{s}^{m} f(x)\right] .
$$

Then from Lemma 3.1, there exists

$$
\mathcal{F}\left(\mathcal{D}_{s}^{\nu} f(x)\right)=(\sigma-i \omega)^{\nu-m} \mathcal{F}\left(D_{s}^{m} f(x)\right)=(\sigma-i \omega)^{\nu} \widehat{f}(\omega),
$$

where $\mathcal{F}\left(D_{s}^{m} f(x)\right)=(\sigma-i \omega)^{m} \widehat{f}(\omega)$ can be proven by the mathematical induction.

In the following, we do the expansions to (1.4) to get the formulas of the coefficients when $p=1,2,3,4,5$; and we prove that the operators have their respective desired convergent order by the technique of Fourier transform.

First, taking $p=1$ and $h$ be the uniform space stepsize, then from (1.4), we have

$$
\kappa^{1, \alpha}(\zeta)=\left(1-\frac{\zeta}{\mathrm{e}^{\sigma h}}\right)^{\alpha}=\sum_{m=0}^{\infty} \mathrm{e}^{-m \sigma h}(-1)^{m}\left(\begin{array}{c}
\alpha \\
m
\end{array}\right) \zeta^{m}=\sum_{m=0}^{\infty} g_{m}^{1, \alpha} \zeta^{m},
$$

with the recursively formula

$$
g_{0}^{1, \alpha}=1, \quad g_{m}^{1, \alpha}=\mathrm{e}^{-\sigma h}\left(1-\frac{\alpha+1}{m}\right) g_{m-1}^{1, \alpha}, \quad m \geq 1,
$$

where $\sigma$ is defined in Definition 1.1.

Similar to the way performed in $[4,5]$, it is easy to compute

$$
\kappa^{p, \alpha}(\zeta)=\left(\sum_{i=1}^{p} \frac{1}{i}\left(1-\frac{\zeta}{\mathrm{e}^{\sigma h}}\right)^{i}\right)^{\alpha}=\sum_{m=0}^{\infty} g_{m}^{p, \alpha} \zeta^{m}, \quad p=1,2,3,4,5,
$$

with $g_{m}^{1, \alpha}$ given in (3.4); and

$$
g_{m}^{p, \alpha}=\mathrm{e}^{-\sigma m h} l_{m}^{p, \alpha}, \quad p=1,2,3,4,5,
$$

where $l_{m}^{1, \alpha}, l_{m}^{2, \alpha}, l_{m}^{3, \alpha}, l_{m}^{4, \alpha}$ and $l_{m}^{5, \alpha}$ are defined by (2.2), (2.4), (2.6), (2.8) and (2.10) in [4], respectively. And it implies that to get the coefficients $g_{m}^{p, \alpha}$, we only need to compute the coefficients $l_{m}^{p, \alpha}$. 
Theorem 3.3. (Case $p=1$ ) Let $f, \mathcal{D}_{s}^{\alpha+1} f(x)$ with $\alpha>0$ and their Fourier transforms belong to $L_{1}(\mathbb{R})$, and denote that

$$
A^{1, \alpha} f(x)=\frac{1}{h^{\alpha}} \sum_{m=0}^{\infty} g_{m}^{1, \alpha} f(x-m h),
$$

where $\mathcal{D}_{s}^{\alpha+1}$ and $g_{m}^{1, \alpha}$ is defined by (3.3) and (3.4), respectively. Then

$$
\mathcal{D}_{s}^{\alpha} f(x)=A^{1, \alpha} f(x)+\mathcal{O}(h) .
$$

Proof. Using Fourier transform, we obtain

$$
\begin{aligned}
\mathcal{F}\left(A^{1, \alpha} f\right)(\omega) & =\frac{1}{h^{\alpha}} \sum_{m=0}^{\infty} g_{m}^{1, \alpha} \mathcal{F}(f(x-m h))(\omega) \\
& =\frac{1}{h^{\alpha}} \sum_{m=0}^{\infty} g_{m}^{1, \alpha}\left(\mathrm{e}^{i \omega h}\right)^{m} \widehat{f}(\omega) \\
& =\frac{1}{h^{\alpha}}\left(1-\frac{\mathrm{e}^{i \omega h}}{\mathrm{e}^{\sigma h}}\right)^{\alpha} \widehat{f}(\omega) \\
& =(\sigma-i \omega)^{\alpha}\left(\frac{1-\mathrm{e}^{-(\sigma-i \omega) h}}{(\sigma-i \omega) h}\right)^{\alpha} \widehat{f}(\omega) \\
& =(\sigma-i \omega)^{\alpha}\left(\frac{1-\mathrm{e}^{-z}}{z}\right)^{\alpha} \widehat{f}(\omega),
\end{aligned}
$$

with $z=(\sigma-i \omega) h$. It is easy to check that

$$
\left(\frac{1-\mathrm{e}^{-z}}{z}\right)^{\alpha}=1-\frac{\alpha}{2} z+\frac{3 \alpha^{2}+\alpha}{24} z^{2}-\frac{\alpha^{3}+\alpha^{2}}{48} z^{3}+\mathcal{O}\left(z^{4}\right) .
$$

Therefore, from Lemma 3.2, there exists

$$
\mathcal{F}\left(A^{1, \alpha} f\right)(\omega)=\mathcal{F}\left(\mathcal{D}_{s}^{\alpha} f\right)+\widehat{\phi}(\omega)
$$

where $\widehat{\phi}(\omega)=(\sigma-i \omega)^{\alpha}\left(-\frac{\alpha}{2} z+\mathcal{O}\left(z^{2}\right)\right) \widehat{f}(\omega), z=(\sigma-i \omega) h$. Then

$$
|\widehat{\phi}(\omega)| \leq \widetilde{c} \cdot\left|(\sigma-i \omega)^{\alpha+1} \widehat{f}(\omega)\right| \cdot h .
$$

With the condition $\mathcal{F}\left[\mathcal{D}_{s}^{\alpha+1} f(x)\right] \in L_{1}(\mathbb{R})$, it leads to

$$
\left|\mathcal{D}_{s}^{\alpha} f(x)-A^{1, \alpha} f(x)\right|=|\phi(x)| \leq \frac{1}{2 \pi} \int_{\mathbb{R}}|\widehat{\phi}(\omega)| d x \leq c|| \mathcal{F}\left[\mathcal{D}_{s}^{\alpha+1} f\right](\omega) \|_{L^{1}} \cdot h=\mathcal{O}(h) .
$$

Theorem 3.4. Let $f, \mathcal{D}_{s}^{\alpha+p} f(x)(p=2,3,4,5)$ with $\alpha>0$ and their Fourier transforms belong to $L_{1}(\mathbb{R})$, and denote that

$$
A^{p, \alpha} f(x)=\frac{1}{h^{\alpha}} \sum_{m=0}^{\infty} g_{m}^{p, \alpha} f(x-m h),
$$

where $g_{m}^{p, \alpha}$ is defined by (3.5). Then

$$
\mathcal{D}_{s}^{\alpha} f(x)=A^{p, \alpha} f(x)+\mathcal{O}\left(h^{p}\right), \quad p=2,3,4,5 .
$$

Proof. Using the ideas of the proof of Theorem 3.3 and Lemmas 2.3-2.7 of [4], we can similarly prove this theorem; the details are omitted here. 
Remark 3.5. Theorems 3.3-3.4 still hold for the fractional substantial integral operators $\mathcal{I}_{s}^{\alpha}$; in fact, comparing Lemmas 3.1 with 3.2 gives us the intuition.

All the above schemes are applicable to finite domain, say, $(a, b)$, after performing zero extensions to the functions considered. Let $f(x)$ be the zero extended function from the finite domain $(a, b)$, and satisfy the requirements of the above corresponding theorems. Taking $p=1,2,3,4,5$ and

$$
\widetilde{A}^{p, \alpha} f(x)=\frac{1}{h^{\alpha}} \sum_{m=0}^{\left[\frac{x-a}{h}\right]} g_{m}^{p, \alpha} f(x-m h), \quad \alpha>0,
$$

with $g_{m}^{p, \alpha}$ given in (3.5). Then

$$
D_{s}^{\alpha} f(x)=\widetilde{A}^{p, \alpha} f(x)+\mathcal{O}\left(h^{p}\right), \quad \alpha>0,
$$

where $D_{s}^{\alpha}$ is defined by (1.2). Thus the approximation operator of (3.6) can be described as

$$
\widetilde{A}^{p, \alpha} f\left(x_{i}\right)=\frac{1}{h^{\alpha}} \sum_{m=0}^{i} g_{m}^{p, \alpha} f\left(x_{i-m}\right), \quad \alpha>0,
$$

where the mesh points $x_{i}=a+i h, i=0, \ldots, M$ and $h=(b-a) / M$ is the uniform space stepsize; the fractional substantial derivative has $p$ th order approximations

$$
D_{s}^{\alpha} f\left(x_{i}\right)=h^{-\alpha} \sum_{m=0}^{i} g_{m}^{p, \alpha} f\left(x_{i-m}\right)+\mathcal{O}\left(h^{p}\right), \quad \alpha>0 .
$$

Similarly, the fractional substantial integral has $p$ th order approximations

$$
I_{s}^{\alpha} f\left(x_{i}\right)=h^{\alpha} \sum_{m=0}^{i} g_{m}^{p,-\alpha} f\left(x_{i-m}\right)+\mathcal{O}\left(h^{p}\right), \quad \alpha>0 .
$$

\section{Discretizations of Fractional substantial CalCulus and its COnVERGence; FRACTIONAL LINEAR MULTISTEP METHODS}

Essentially the results given this section are the generalizations of the ones for fractional calculus provided in [11] to fractional substantial calculus; some of them are not straightforward, so we restate and prove them. In particular, comparing with Section 3, by adding some terms at the neighborhood of the boundary of the fractional substantial calculus, we can relax the regularity requirements of the performed functions but still preserve the desired convergent order.

For the simplicity of presentation, we take the lower terminal $a=0$ (that is not essential, $a$ can be any given constant but not infinity). Then the fractional substantial integral (1.1) and fractional substantial derivative (1.2), respectively, reduce to

$$
I_{s}^{\alpha} f(x)=\frac{1}{\Gamma(\alpha)} \int_{0}^{x}(x-\tau)^{\alpha-1} \mathrm{e}^{-\sigma(x-\tau)} f(\tau) \mathrm{d} \tau
$$

and

$$
D_{s}^{\alpha} f(x)=D_{s}^{m}\left[I_{s}^{m-\alpha} f(x)\right]
$$

where $m$ is the smallest integer that exceeds $\alpha$. 
For $\sigma=0$, the fractional substantial integral (4.1) and fractional substantial derivative (4.2), respectively, reduce to the Riemann-Liouville fractional integral

$$
I^{\alpha} f(x)=\frac{1}{\Gamma(\alpha)} \int_{0}^{x}(x-\tau)^{\alpha-1} f(\tau) \mathrm{d} \tau,
$$

and Riemann-Liouville fractional derivative $[9,15,16]$

$$
D^{\alpha} f(x)=\frac{\mathrm{d}^{m}}{\mathrm{~d} x^{m}} \frac{1}{\Gamma(m-\alpha)} \int_{0}^{x}(x-\tau)^{m-\alpha-1} f(\tau) d \tau, \quad m-1<\alpha<m .
$$

Using the homogeneity and the convolution structure of $I^{\alpha}$ in (4.3):

$$
\left(I^{\alpha} f\right)(x)=x^{\alpha}\left(I^{\alpha} f(t x)\right)(1) \text { and } I^{\alpha} f=\frac{1}{\Gamma(\alpha)} t^{\alpha-1} * f,
$$

Lubich gets the following important property [11]

$$
\left(E_{h}^{\alpha} t^{\beta-1}\right)(x)=x^{\alpha+\beta-1}\left(E_{h / x}^{\alpha} t^{\beta-1}\right)(1) ; \quad\left(E_{h}^{\alpha} t^{\beta-1}\right)(1)=\mathcal{O}\left(h^{\beta}\right)+\mathcal{O}\left(h^{p}\right)
$$

and

$I^{\alpha}(f * g)=\frac{1}{\Gamma(\alpha)} t^{\alpha-1} *(f * g)=\left(\frac{1}{\Gamma(\alpha)} t^{\alpha-1} * f\right) * g=\left(I^{\alpha} f\right) * g ; \quad \Omega_{h}^{\alpha}(f * g)=\left(\Omega_{h}^{\alpha} f\right) * g ; \quad E_{h}^{\alpha}(f * g)=\left(E_{h}^{\alpha} f\right) * g$,

where

$$
E_{h}^{\alpha}=\Omega_{h}^{\alpha}-I^{\alpha} \text { and } \Omega_{h}^{\alpha} f(x)=h^{\alpha} \sum_{j=0}^{n} \omega_{n-j}^{\alpha} f(j h), \quad(x=n h),
$$

and $\omega_{n}^{\alpha}$ denotes the convolution quadrature weights. So Lubich obtains the following convolution quadratures to approximation the Riemann-Liouville fractional integral

$$
I_{h}^{\alpha} f(x)=h^{\alpha} \sum_{j=0}^{n} \omega_{n-j}^{\alpha} f(j h)+h^{\alpha} \sum_{j=1}^{r} \omega_{n, j}^{\alpha} f(j h), \quad(x=n h), \quad \alpha>0,
$$

where $\omega_{n, j}^{\alpha}$ denotes the starting quadrature weights. The added term $h^{\alpha} \sum_{j=1}^{r} \omega_{n, j}^{\alpha} f(j h)$ is mainly for keeping the accuracy when relaxing the requirement of the regularity of $f(x)$.

For $D_{h}^{\alpha} f(x)$ or $I_{h}^{-\alpha} f(x)$ in (4.6) with $\alpha>0$, taking $D^{(j)} f(0)=0, j=0,1, \ldots, m-1, m-1<\alpha<m$, then it yields the convolution structure of $D^{\alpha}$ in (4.4):

$$
\begin{aligned}
D^{\alpha} f(x) & =\frac{\mathrm{d}^{m}}{\mathrm{~d} x^{m}}\left[\frac{1}{\Gamma(m-\alpha)} \int_{0}^{x}(x-\tau)^{m-\alpha-1} f(\tau) \mathrm{d} \tau\right] \\
& =\frac{1}{\Gamma(m-\alpha)} \int_{0}^{x}(x-\tau)^{m-\alpha-1}\left(\mathrm{~d}^{m} f(\tau) / \mathrm{d} \tau^{m}\right) \mathrm{d} \tau=\frac{1}{\Gamma(m-\alpha)} x^{m-\alpha-1} * \frac{\mathrm{d}^{m} f(x)}{\mathrm{d} x^{m}},
\end{aligned}
$$

and the homogeneity of $D^{\alpha}$ :

$$
\left(D^{\alpha} f\right)(x)=x^{m-\alpha}\left(D^{\alpha} f(t x)\right)(1), \quad m-1<\alpha<m .
$$

Therefore, we also obtain following property

$$
\left(E_{h}^{-\alpha} t^{\beta-1}\right)(x)=x^{-\alpha+\beta-1}\left(E_{h / x}^{-\alpha} t^{\beta-1}\right)(1), \quad \beta>m,
$$


where

$$
E_{h}^{-\alpha}=\Omega_{h}^{-\alpha}-D^{\alpha} \text { and } \Omega_{h}^{-\alpha} f(x)=h^{-\alpha} \sum_{j=0}^{n} \omega_{n-j}^{-\alpha} f(j h), \quad(x=n h) .
$$

So similar to the discussions in [11], we can also get the following scheme to approximate the RiemannLiouville fractional derivative

$$
D_{h}^{\alpha} f(x)=h^{-\alpha} \sum_{j=0}^{n} \omega_{n-j}^{-\alpha} f(j h)+h^{-\alpha} \sum_{j=1}^{r} \omega_{n, j}^{-\alpha} f(j h), \quad(x=n h), \quad \alpha>0 .
$$

Form (4.6) and (4.7), there exists

$$
I_{h}^{\alpha} f(x)=h^{\alpha} \sum_{j=0}^{n} \omega_{n-j} f(j h)+h^{\alpha} \sum_{j=1}^{r} \omega_{n, j} f(j h), \quad(x=n h), \quad \alpha \in \mathbb{R},
$$

where $\alpha>0$ corresponds to (4.6) $\left(\omega_{n}=\omega_{n}^{\alpha}, \omega_{n, j}=\omega_{n, j}^{\alpha}\right)$ and $\alpha<0$ corresponds to (4.7) $\left(\omega_{n}=\omega_{n}^{-\alpha}, \omega_{n, j}=\right.$ $\left.\omega_{n, j}^{-\alpha}\right)$.

In this section, we mainly focus on the discretized fractional substantial calculus; for simplicity, the following notations are used:

$$
E_{s, h}^{\alpha}=\Omega_{s, h}^{\alpha}-I_{s}^{\alpha}, \quad \text { where } \Omega_{s, h}^{\alpha} f(x)=h^{\alpha} \sum_{j=0}^{n} \kappa_{n-j} f(j h), \quad(x=n h), \quad \alpha \in \mathbb{R},
$$

and it is easy to get the following properties for $\alpha \in \mathbb{R}$ :

$$
\begin{aligned}
\left(I_{s}^{\alpha}\left[\mathrm{e}^{-\sigma t} f(t)\right]\right)(x) & =\mathrm{e}^{-\sigma x}\left(I^{\alpha}[f(t)]\right)(x) ; \\
\left(E_{s, h}^{\alpha}\left[\mathrm{e}^{-\sigma t} f(t)\right]\right)(x) & =\mathrm{e}^{-\sigma x}\left(E_{h}^{\alpha}[f(t)]\right)(x),
\end{aligned}
$$

and

$$
I_{s}^{\alpha}(f * g)=\left(I_{s}^{\alpha} f\right) * g ; \quad \Omega_{s, h}^{\alpha}(f * g)=\left(\Omega_{s, h}^{\alpha} f\right) * g ; \quad E_{s, h}^{\alpha}(f * g)=\left(E_{s, h}^{\alpha} f\right) * g,
$$

where $\alpha>0$ corresponds to fractional substantial integral and $\alpha<0$ corresponds to fractional substantial derivative.

So we consider the following scheme to approximate the fractional substantial integral (4.1) or the fractional substantial derivative (4.2)

$$
I_{s, h}^{\alpha} f(x)=h^{\alpha} \sum_{j=0}^{n} \kappa_{n-j}^{p, \alpha} f(j h)+h^{\alpha} \sum_{j=1}^{r} \kappa_{n, j} f(j h), \quad(x=n h), \quad \alpha \in \mathbb{R},
$$

where

$$
\kappa_{j}^{p, \alpha}=\mathrm{e}^{-j \sigma h} \omega_{j}, \quad \omega_{j} \text { is defined by (4.8), }
$$

and $\kappa_{j}^{p, \alpha}$ (being simply denoted as $\kappa_{j}$ hereafter) and $\kappa_{n, j}$ also denote the convolution quadrature weights and the starting quadrature weights, respectively.

Given a sequence $\kappa=\left(\kappa_{n}\right)_{0}^{\infty}\left(\right.$ or $\left.\omega=\left(\omega_{n}\right)_{0}^{\infty}\right)$ and take [11]

$$
\kappa(\zeta)=\sum_{n=0}^{\infty} \kappa_{n} \zeta^{n}, \quad\left(\text { or } \omega(\zeta)=\sum_{n=0}^{\infty} \omega_{n} \zeta^{n}\right),
$$

to be its generating power series. 
Definition 4.1. A convolution quadrature $\kappa$ is stable (for $I_{s}^{\alpha}$ ) if

$$
\kappa_{n}=\mathcal{O}\left(n^{\alpha-1}\right)
$$

Definition 4.2. A convolution quadrature $\kappa$ is consistent of order $p$ (for $I_{s}^{\alpha}$ ) if

$$
h^{\alpha} \kappa\left(\mathrm{e}^{\sigma h} \mathrm{e}^{-h}\right)=1+\mathcal{O}\left(h^{p}\right) .
$$

Definition 4.3. A convolution quadrature $\kappa$ is convergent of order $p\left(\right.$ to $\left.I_{s}^{\alpha}\right)$ if

$$
\left(E_{s, h}^{\alpha}\left[\mathrm{e}^{-\sigma t} t^{\beta-1}\right]\right)(1)=\mathcal{O}\left(h^{\beta}\right)+\mathcal{O}\left(h^{p}\right) \text { for all } \beta \in \mathbb{C}, \beta \neq 0,-1,-2, \ldots
$$

Lemma 4.4. If $\left(E_{s, h}^{\alpha}\left[\mathrm{e}^{-\sigma t} t^{k-1}\right]\right)(1)=\mathcal{O}\left(h^{k}\right)+\mathcal{O}\left(h^{p}\right)$ for $k=1,2,3, \ldots$, then $\kappa$ is consistent of order $p$. Moreover, $\kappa$ is consistent of order $p$ if and only if $\omega$ is consistent of order $p$.

Proof. According to (4.10), we have

$$
\left(E_{s, h}^{\alpha}\left[\mathrm{e}^{-\sigma t} t^{k-1}\right]\right)(1)=\mathrm{e}^{-\sigma}\left(E_{h}^{\alpha} t^{k-1}\right)(1),
$$

and it leads to

$$
\left(E_{h}^{\alpha} t^{k-1}\right)(1)=\mathcal{O}\left(h^{k}\right)+\mathcal{O}\left(h^{p}\right), \text { for } k=1,2,3, \ldots
$$

Then from Lemma 3.1 of [11], we obtain

$$
h^{\alpha} \omega\left(\mathrm{e}^{-h}\right)=1+\mathcal{O}\left(h^{p}\right), \text { with } \omega(\zeta)=\sum_{n=0}^{\infty} \omega_{n} \zeta^{n} .
$$

Using (4.13), there exists

$$
\kappa(\zeta)=\sum_{n=0}^{\infty} \kappa_{n} \zeta^{n}=\sum_{n=0}^{\infty} \mathrm{e}^{-n \sigma h} \omega_{n} \zeta^{n}=\omega\left(\frac{\zeta}{\mathrm{e}^{\sigma h}}\right) .
$$

Therefore

$$
h^{\alpha} \kappa\left(\mathrm{e}^{\sigma h} \mathrm{e}^{-h}\right)=h^{\alpha} \omega\left(\mathrm{e}^{-h}\right)=1+\mathcal{O}\left(h^{p}\right),
$$

and it means that $\kappa$ is consistent of order $p$ if and only if $\omega$ is consistent of order $p$.

Using (3.6) of [11] and (4.15), we get

$$
\begin{aligned}
\kappa(\zeta)=\omega\left(\frac{\zeta}{\mathrm{e}^{\sigma h}}\right)= & \left(1-\frac{\zeta}{\mathrm{e}^{\sigma h}}\right)^{-\alpha}\left[c_{0}+c_{1}\left(1-\frac{\zeta}{\mathrm{e}^{\sigma h}}\right)+c_{2}\left(1-\frac{\zeta}{\mathrm{e}^{\sigma h}}\right)^{2}+\ldots\right. \\
& \left.+c_{N-1}\left(1-\frac{\zeta}{\mathrm{e}^{\sigma h}}\right)^{N-1}+\left(1-\frac{\zeta}{\mathrm{e}^{\sigma h}}\right)^{N} \widetilde{r}\left(\frac{\zeta}{\mathrm{e}^{\sigma h}}\right)\right]
\end{aligned}
$$

and

$$
\kappa(\zeta)=\omega\left(\frac{\zeta}{\mathrm{e}^{\sigma h}}\right)=\left(1-\frac{\zeta}{\mathrm{e}^{\sigma h}}\right)^{-\alpha} \widetilde{\omega}\left(\frac{\zeta}{\mathrm{e}^{\sigma h}}\right) .
$$

Therefore, we can characterize consistency in terms of the coefficients $c_{i}$.

Lemma 4.5. Let $\sum_{i=0}^{\infty} \gamma_{i}(1-\zeta)^{i}=\left(-\frac{\ln \zeta}{1-\zeta}\right)^{-\alpha}$. Then $\kappa$ is consistent of order $p$ if and only if the coefficients $c_{i}$ in (4.16) satisfy

$$
c_{i}=\gamma_{i} \text { for } i=0,1, \ldots, p-1 \text {. }
$$


Proof. From Lemma 4.4, it implies that $\kappa$ is consistent of order $p$ if and only if $\omega$ is consistent of order $p$. Thus, using Lemma 3.2 of [11], the desired result is obtained.

Whether the method $\kappa$ is stable depends on the remainder in the expansion (4.16), and (4.16) can be rewritten as

$$
\begin{aligned}
\kappa(\zeta)= & \left(1-\frac{\zeta}{\mathrm{e}^{\sigma h}}\right)^{-\alpha}\left[c_{0}+c_{1}\left(1-\frac{\zeta}{\mathrm{e}^{\sigma h}}\right)+\ldots+c_{N-1}\left(1-\frac{\zeta}{\mathrm{e}^{\sigma h}}\right)^{N-1}\right] \\
& +\left(1-\frac{\zeta}{\mathrm{e}^{\sigma h}}\right)^{N} r\left(\frac{\zeta}{\mathrm{e}^{\sigma h}}\right),
\end{aligned}
$$

where $r(\zeta)=(1-\zeta)^{-\alpha} \widetilde{r}(\zeta)$.

Lemma 4.6. $\kappa$ is stable if and only if $\omega$ is stable; and $\omega$ is stable if and only if the coefficients $r_{n}$ of $r(\zeta)$ in (4.17) satisfy

$$
r_{n}=\mathcal{O}\left(n^{\alpha-1}\right) .
$$

Proof. By Lemma 3.3 of [11], we have $\omega$ is stable if and only if $r_{n}=\mathcal{O}\left(n^{\alpha-1}\right)$. From (4.13) and $\mathrm{e}^{-j \sigma h} \in$ $\left[\mathrm{e}^{-|\sigma| x}, \mathrm{e}^{|\sigma| x}\right], j=0,1, \ldots, n, x=n h$, it implies that $\kappa$ is stable if and only if $\omega$ is stable.

Lemma 4.7. Convergence implies stability. Moreover, $\kappa$ is convergent of order $p$ if and only if $\omega$ is convergent of order $p$.

Proof. According to (4.10), we have

$$
\left(E_{s, h}^{\alpha}\left[\mathrm{e}^{-\sigma t} t^{\beta-1}\right]\right)(1)=\mathrm{e}^{-\sigma}\left(E_{h}^{\alpha} t^{\beta-1}\right)(1),
$$

and it implies that $\kappa$ is convergent of order $p$ if and only if $\omega$ is convergent of order $p$. Hence, according to Lemma 3.4 of [11], the desired result is got.

Lemma 4.8. Let $\alpha, \beta \in \mathbb{C}, \beta \neq 0,-1,-2, \cdots$. If $\kappa$ is stable, then the convolution quadrature error of $\mathrm{e}^{-\sigma t} t^{\beta-1}$ has the asymptotic expansion as

$$
\left(E_{s, h}^{\alpha}\left[\mathrm{e}^{-\sigma t} t^{\beta-1}\right]\right)(1)=\mathrm{e}^{-\sigma}\left(e_{0}+e_{1} h+\cdots+e_{N-1} h^{N-1}+\mathcal{O}\left(h^{N}\right)+\mathcal{O}\left(h^{\beta}\right)\right),
$$

and the coefficients $e_{j}=e_{j}\left(\alpha, \beta, c_{0}, \ldots, c_{j}\right)$ depend analytically on $\alpha, \beta$ and the coefficients $c_{0}, \ldots, c_{j}$ of (4.17).

Proof. From Lemma 4.6, $\kappa$ is stable if and only if $\omega$ is stable. According to (4.10) and Lemma 3.5 of [11], we get

$$
\begin{aligned}
\left(E_{s, h}^{\alpha}\left[\mathrm{e}^{-\sigma t} t^{\beta-1}\right]\right)(1) & =\mathrm{e}^{-\sigma}\left(E_{h}^{\alpha} t^{\beta-1}\right)(1) \\
& =\mathrm{e}^{-\sigma}\left(e_{0}+e_{1} h+\cdots+e_{N-1} h^{N-1}+\mathcal{O}\left(h^{N}\right)+\mathcal{O}\left(h^{\beta}\right)\right) .
\end{aligned}
$$

Lemma 4.9. Let $\Re(\alpha)>0$. If $\left(E_{s, h}^{\alpha}\left[\mathrm{e}^{-\sigma t} t^{p-1}\right]\right)(1)=\mathcal{O}\left(h^{p}\right)$, then $\left(E_{s, h}^{\alpha}\left[\mathrm{e}^{-\sigma t} t^{\beta-1}\right]\right)(1)=\mathcal{O}\left(h^{p}\right)$ for all $\Re(\beta)>p$. Proof. According to (4.10), it leads to

$$
\left(E_{s, h}^{\alpha}\left[\mathrm{e}^{\sigma t} t^{p-1}\right]\right)(1)=\mathrm{e}^{-\sigma}\left(E_{h}^{\alpha} t^{p-1}\right)(1)=\mathcal{O}\left(h^{p}\right)
$$

Then form Lemma 3.6 of [11], we obtain $\left(E_{h}^{\alpha} t^{\beta-1}\right)(1)=\mathcal{O}\left(h^{p}\right)$ for all $\Re(\beta)>p$. Using (4.10) again, there exists $\left(E_{s, h}^{\alpha}\left[\mathrm{e}^{-\sigma t} t^{\beta-1}\right]\right)(1)=\mathcal{O}\left(h^{p}\right)$ for all $\Re(\beta)>p$. 
Lemma 4.10. Let $\Re(\alpha)>0$. There exist $\widetilde{\gamma}_{0}, \widetilde{\gamma}_{1}, \ldots$ (independent of $\kappa$ ) such that the following holds for stable $\kappa$ :

$$
\left(E_{s, h}^{\alpha}\left[\mathrm{e}^{-\sigma t} t^{q-1}\right]\right)(1)=\mathcal{O}\left(h^{q}\right), \text { for } q=1,2, \ldots, p,
$$

if and only if $c_{i}$ of (4.17) satisfy

$$
c_{i}=\widetilde{\gamma}_{i}, \text { for } i=0,1, \ldots, p-1 .
$$

Proof. From Lemma 4.6, $\kappa$ is stable if and only if $\omega$ is stable. From (4.10) and Lemma 3.7 of [11], there eixsts

$$
\left(E_{s, h}^{\alpha}\left[\mathrm{e}^{-\sigma t} t^{q-1}\right]\right)(1)=\mathrm{e}^{-\sigma}\left(E_{h}^{\alpha} t^{q-1}\right)(1)=\mathcal{O}\left(h^{q}\right),
$$

if and only if

$$
\left(E_{h}^{\alpha} t^{q-1}\right)(1)=\mathcal{O}\left(h^{q}\right), \text { for } q=1,2, \ldots, p,
$$

if and only if the coefficients $c_{i}$ of (4.17) satisfy

$$
c_{i}=\widetilde{\gamma}_{i}, \text { for } i=0,1, \ldots, p-1 .
$$

Lemma 4.11. Let $\alpha \in \mathbb{R} . \kappa$ is convergent of order $p$, if it is stable and consistent of order $p$.

Proof. According to Lemmas 4.6 and 4.4, $\kappa$ is stable and consistent of order $p$ if and only if $\omega$ is stable and consistent of order $p$. Then from Lemma 3.8 of [11], $\omega$ is convergent of order $p$, and it leads to that $\kappa$ is also convergent of order $p$ by Lemmas 4.7 .

Theorem 4.12. $\kappa$ is stable and consistent of order $p$ if and only if it is convergent of order $p$.

Proof. From lemmas 4.4, 4.7 and 4.11, we obtain it.

Theorem 4.13. Let $\kappa$ satisfy (4.14), and $f(x)=x^{\beta-1} g(x)$, where $\beta \neq 0,-1,-2, \ldots$, for $\alpha \geq 0$ and $\beta>\lceil-\alpha\rceil$ for $\alpha<0$; and $g(x)$ is sufficiently differentiable. Then, there exists a starting quadrature $\kappa_{n, j}$, such that the approximation $I_{s, h}^{\alpha} f$ given by (4.12) satisfies

$$
I_{s, h}^{\alpha} f(x)-I_{s}^{\alpha} f(x)=\mathcal{O}\left(h^{p}\right) .
$$

Proof. A suitable starting quadrature can be chosen by putting

$$
I_{s, h}^{\alpha}\left[\mathrm{e}^{-\sigma t} t^{q+\beta-1}\right](x)-I_{s}^{\alpha}\left[\mathrm{e}^{-\sigma t} t^{q+\beta-1}\right](x)=0, \quad q=0,1, \ldots, m,
$$

where $m$ satisfies $\Re(m+\beta-1) \leq p<\Re(m+\beta)$; then the following holds

$$
h^{\alpha} \sum_{j=1}^{m+1} \kappa_{n, j} \mathrm{e}^{-\sigma j h}(j h)^{q+\beta-1}+\left(E_{s, h}^{\alpha}\left[\mathrm{e}^{-\sigma t} t^{q+\beta-1}\right]\right)(1)=0, \quad n h=1 .
$$

From (4.14) and (4.18), we have

$$
\sum_{j=1}^{m+1} \kappa_{n, j} \mathrm{e}^{-\sigma j h} j^{q+\beta-1}=\mathcal{O}\left(h^{1-\alpha}\right)+\mathcal{O}\left(h^{p-\alpha-q-\beta+1}\right), \quad q=0,1, \ldots, m .
$$

Using (4.18) again, we have

$$
\sum_{j=1}^{m+1} \kappa_{n, j} \mathrm{e}^{-\sigma j h} j^{q+\beta-1}=\frac{\Gamma(q+\beta)}{\Gamma(\alpha+q+\beta)} \mathrm{e}^{-\sigma n h} n^{q+\alpha+\beta-1}-\sum_{j=1}^{n} \kappa_{n-j} \mathrm{e}^{-\sigma j h} j^{q+\beta-1},
$$


i.e.,

$$
\left[\begin{array}{llll}
\mathrm{e}^{-\sigma h} 1^{\beta-1} & \mathrm{e}^{-2 \sigma h} 2^{\beta-1} & \cdots & \mathrm{e}^{-(m+1) \sigma h}(m+1)^{\beta-1} \\
\mathrm{e}^{-\sigma h} 1^{\beta} & \mathrm{e}^{-2 \sigma h} 2^{\beta} & \cdots & \mathrm{e}^{-(m+1) \sigma h}(m+1)^{\beta} \\
\vdots & \vdots & \cdots & \vdots \\
\mathrm{e}^{-\sigma h} 1^{\beta+m-1} & \mathrm{e}^{-2 \sigma h} 2^{\beta+m-1} & \cdots & \mathrm{e}^{-(m+1) \sigma h}(m+1)^{\beta+m-1}
\end{array}\right]\left[\begin{array}{l}
\kappa_{n, 1} \\
\kappa_{n, 2} \\
\vdots \\
\kappa_{n, m+1}
\end{array}\right]=\left[\begin{array}{c}
F_{0} \\
F_{1} \\
\vdots \\
F_{m}
\end{array}\right],
$$

where $F_{q}=\frac{\Gamma(q+\beta)}{\Gamma(\alpha+q+\beta)} \mathrm{e}^{-\sigma n h} n^{q+\alpha+\beta-1}-\sum_{j=1}^{n} \kappa_{n-j} \mathrm{e}^{-\sigma j h} j^{q+\beta-1}$; this gives a Vandermonde type system for $\kappa_{n, j}$, and it becomes the Vandermonde system when $\sigma=0$.

Let $f(x)=x^{\beta-1} g(x)=\mathrm{e}^{-\sigma x} x^{\beta-1} h(x)$, where $h(x)=\mathrm{e}^{\sigma x} g(x)$, and $g(x)$ is sufficiently differentiable. From Lemma 2.7, there exists

$$
\begin{aligned}
f(x) & =\sum_{q=0}^{m} \frac{D_{s}^{(q+\beta-1)} f(0)}{\Gamma(q+\beta)} x^{q+\beta-1} \mathrm{e}^{-\sigma x}+\frac{1}{\Gamma(m+\beta)}\left[\left(t^{m+\beta-1} \mathrm{e}^{-\sigma t}\right) * D_{s}^{(m+\beta)} f\right](x) \\
& :=A(x)+B(x) .
\end{aligned}
$$

From (4.5) and (4.10), we obtain

$$
\begin{aligned}
\left(E_{s, h}^{\alpha}\left[\mathrm{e}^{-\sigma t} t^{m+\beta-1}\right]\right)(x) & =\mathrm{e}^{-\sigma x}\left(E_{h}^{\alpha}\left[t^{m+\beta-1}\right]\right)(x)=\mathrm{e}^{-\sigma x} x^{\alpha+m+\beta-1}\left(E_{h / x}^{\alpha}\left[t^{m+\beta-1}\right]\right)(1) \\
& =\mathcal{O}\left(\mathrm{e}^{-\sigma x} x^{\alpha+m+\beta-1-p} h^{p}\right) .
\end{aligned}
$$

Then utilizing (4.11) and the boundedness of $D_{s}^{(m+\beta)}$, we have

$$
\begin{aligned}
\left(E_{s, h}^{\alpha}\left[\left(t^{m+\beta-1} \mathrm{e}^{-\sigma t}\right) * D_{s}^{(m+\beta)} f\right]\right)(x) & =\left(\left[E_{s, h}^{\alpha}\left(t^{m+\beta-1} \mathrm{e}^{-\sigma t}\right)\right] * D_{s}^{(m+\beta)} f\right)(x) \\
& =\mathcal{O}\left(\mathrm{e}^{-\sigma x} x^{\alpha+m+\beta-p} h^{p}\right) .
\end{aligned}
$$

According to (4.18), (4.19), (4.21), and (4.22), there exists

$$
\begin{aligned}
I_{s, h}^{\alpha} f(x)-I_{s}^{\alpha} f(x) & =E_{s, h}^{\alpha} f(x)-h^{\alpha} \sum_{j=1}^{m+1} \kappa_{n, j} f(j h) \\
& =E_{s, h}^{\alpha} B(x)-h^{\alpha} \sum_{j=1}^{m+1} \kappa_{n, j} B(j h) \\
& =\mathcal{O}\left(\mathrm{e}^{-\sigma x} x^{\alpha+m+\beta-p} h^{p}\right)+\mathcal{O}\left(h^{\alpha+m+\beta-1}\right) \sum_{j=1}^{m+1} \kappa_{n, j} \mathrm{e}^{-\sigma j h} j^{m+\beta-1} \\
& =\mathcal{O}\left(h^{p}\right) \text { uniformly for bounded } x .
\end{aligned}
$$

Remark 4.14. When computing the fractional substantial derivative or integral of a given function $f(x)$ defined in $[a,+\infty)$, we prefer the first method (3.8) if $f(x)$ itself and its several derivatives (from 1 to $p-1$ ) equal to zero at the point $a$, otherwise we choose the second one (4.12).

\section{Numerical RESUlTS}

We use two numerical examples to confirm the theoretical results given in the above sections, including the fractional substantial derivatives and integrals. The first example mainly verifies the truncation errors; the second one primarily focuses on illustrating that the starting quadrature numerically works very well for keeping the high order accuracy when the performed function becomes less regular. The third example shows that the shifted scheme can effectively solve a two-point boundary value problem. And the $l_{\infty}$ norm is used to measure the numerical errors. 
TABLE 2. The maximum errors and convergence orders for (3.8), when $p=5, \sigma=1 / 2$.

\begin{tabular}{lcccccc}
\hline$h$ & $\alpha=-0.5$ & Rate & $\alpha=0.5$ & Rate & $\alpha=1.5$ & Rate \\
\hline $1 / 10$ & $3.7956 \mathrm{e}-005$ & & $2.0214 \mathrm{e}-004$ & & $3.7954 \mathrm{e}-003$ & \\
$1 / 20$ & $1.3109 \mathrm{e}-006$ & 4.8557 & $6.9814 \mathrm{e}-006$ & 4.8557 & $1.2933 \mathrm{e}-004$ & 4.8751 \\
$1 / 40$ & $4.3065 \mathrm{e}-008$ & 4.9279 & $2.2935 \mathrm{e}-007$ & 4.9279 & $4.3193 \mathrm{e}-006$ & 4.9041 \\
$1 / 80$ & $1.3798 \mathrm{e}-009$ & 4.9639 & $7.3488 \mathrm{e}-009$ & 4.9639 & $1.4014 \mathrm{e}-007$ & 4.9459 \\
\hline
\end{tabular}

TABLE 3. The maximum errors and convergence orders for (4.12) and (3.8), respectively, when $p=5, \sigma=1 / 2, \beta=1.6, r=4$.

\begin{tabular}{|c|c|c|c|c|c|c|c|c|}
\hline \multicolumn{5}{|c|}{ Numerical scheme (4.12) } & \multicolumn{4}{|c|}{ Numerical scheme (3.8) } \\
\hline$h$ & $\alpha=-0.5$ & Rate & $\alpha=0.5$ & Rate & $\alpha=-0.5$ & Rate & $\alpha=0.5$ & Rate \\
\hline $1 / 10$ & $2.8710 \mathrm{e}-05$ & & $3.7035 \mathrm{e}-04$ & & $1.4508 \mathrm{e}-02$ & & $4.3208 \mathrm{e}-01$ & \\
\hline $1 / 20$ & $1.0424 \mathrm{e}-06$ & 4.78 & $1.2791 \mathrm{e}-05$ & 4.86 & $6.9407 \mathrm{e}-03$ & 1.06 & $4.1336 \mathrm{e}-01$ & 0.064 \\
\hline $1 / 40$ & $3.5111 \mathrm{e}-08$ & 4.90 & $4.2020 \mathrm{e}-07$ & 4.93 & $3.2787 \mathrm{e}-03$ & 1.08 & $3.9053 \mathrm{e}-01$ & 0.082 \\
\hline $1 / 80$ & $1.1391 \mathrm{e}-09$ & 4.95 & $1.3464 \mathrm{e}-08$ & 4.96 & $1.5392 \mathrm{e}-03$ & 1.09 & $3.6666 \mathrm{e}-01$ & 0.091 \\
\hline $1 / 160$ & $3.6272 \mathrm{e}-11$ & 4.97 & $4.2604 \mathrm{e}-10$ & 4.98 & $7.2029 \mathrm{e}-04$ & 1.10 & $3.4318 \mathrm{e}-01$ & 0.096 \\
\hline
\end{tabular}

Example 5.1. To numerically verify the truncation error given in Theorem 3.4 in a bounded domain, we utilize the approximation (3.8) with $p=5$ to compute $D_{s}^{\alpha} f(x)$, where $f(x)=\mathrm{e}^{-\sigma x} x^{5+\alpha}$ with $x \in(0,1)$ and $\sigma$ is taken as $\frac{1}{2}$; and by comparing with the analytical solution $\frac{\Gamma(6+\alpha)}{\Gamma(6)} x^{5} \mathrm{e}^{-\sigma x}$, we get the numerical errors and convergence orders in Table 2 .

Table 2 numerically verifies Theorem 3.4, and shows that the truncation errors are $\mathcal{O}\left(h^{5}\right)$.

Example 5.2. To numerically confirm the result given in Section 4 that the starting quadrature can keep the accuracy when the performed function is not sufficiently regular, we utilize the approximation (4.12) and (3.8) (both with $p=5$ ), respectively, to simulate $D_{s}^{\alpha} f(x)$, where $f(x)=\mathrm{e}^{-\sigma x}\left(x^{5+\alpha}+x^{0.6}\right)$ with $x \in(0,1)$ and $\sigma$ is taken as $\frac{1}{2}$; and by comparing with the analytical solution $\frac{\Gamma(6+\alpha)}{\Gamma(6)} x^{5} \mathrm{e}^{-\sigma x}+\frac{\Gamma(1.6)}{\Gamma(1.6-\alpha)} x^{0.6-\alpha} \mathrm{e}^{-\sigma x}$, we get the numerical errors and convergence orders in Table 3.

Table 3 numerically verifies Theorem 4.13, i.e., when the performed functions are less regular, the scheme (4.12) still keeps the high convergence order but the scheme (3.8) fails.

Example 5.3. By shifting (3.8), we simulate the following problem

$$
-D_{s}^{\alpha} y(x)+y(x)=0, \quad \alpha \in(1,2),
$$

on a finite domain $0<x<1$; and take $\sigma=\frac{1}{2}, h=\frac{1}{50}$, and the boundary conditions $y(0)=y(1)=1$. Here, the shifted difference operator [19] is defined by

$$
D_{s, h}^{\alpha} y\left(x_{i}\right)=\frac{1}{h^{\alpha}} \sum_{k=0}^{i+1} g_{k}^{p, \alpha} y\left(x_{i-k+1}\right) \quad \text { with } p=1 .
$$

Figure 1 shows the simulation results, which confirm the effectiveness of the scheme. Besides this, for more complex simulations see [6].

\section{Conclusions}

When studying the anomalous diffusion, CTRW is the most widely used model. However, if the boundary conditions and external fields are involved, the equations are more convenient to include these quantities. Assuming the probability density functions (PDFs) of the waiting time and jump lengths in CTRW model 


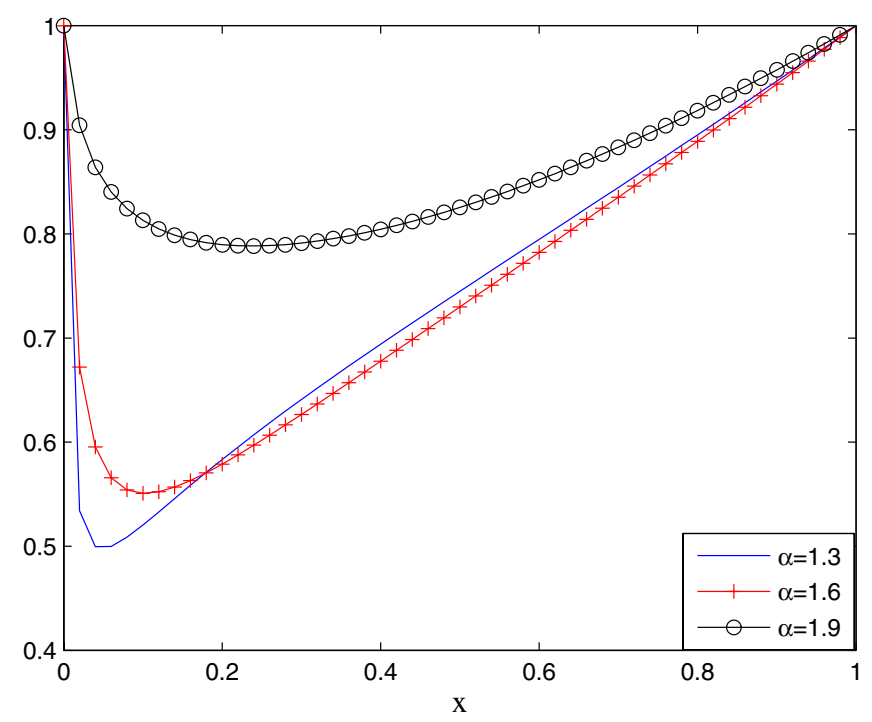

Figure 1. Simulations to (5.1).

are independent, from CTRW model we can derive the corresponding fractional partial differential equations (PDEs). When extending the concept of CTRW to position-velocity space, the derived PDEs usually have a fractional substantial derivative/integral [8]. Nowadays, it seems that there are less mathematical works for these kind of operators. This paper detailedly discusses the properties of fractional substantial calculus, and provides a series of high order discretization schemes, which can be used to effectively solve PDEs with fractional substantial calculus [6].

\section{Appendixes}

We provide the pseudo codes used in this paper. According to Remark 4.14, one can choose Algorithm 1 or 2 to compute fractional substantial derivative/integral of a given function.
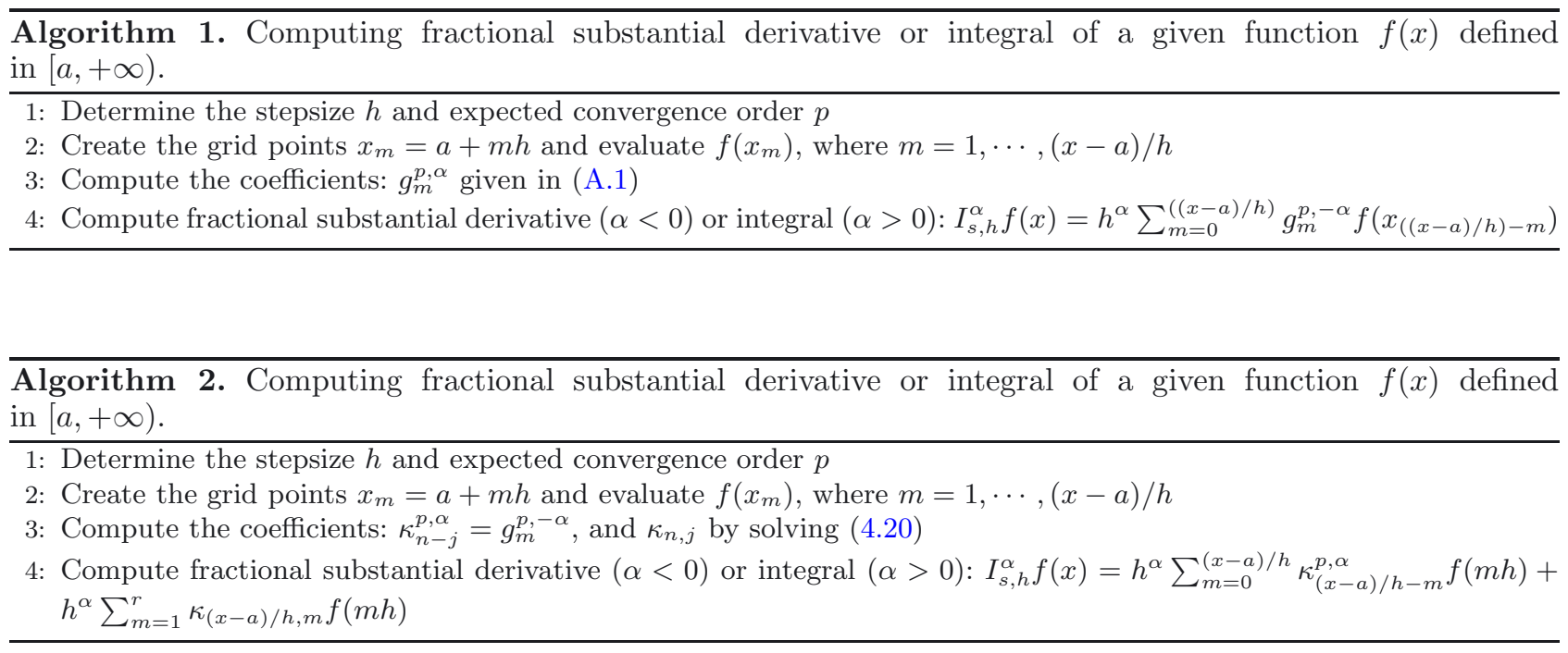
The coefficients used in Algorithms 1-2 [4]:

$$
g_{m}^{p, \alpha}=\mathrm{e}^{-\sigma m h} l_{m}^{p, \alpha}, \quad p=1,2,3,4,5,
$$

where

$$
\begin{aligned}
& l_{0}^{1, \alpha}=1, \quad l_{m}^{1, \alpha}=\left(1-\frac{\alpha+1}{m}\right) l_{m-1}^{1, \alpha}, \quad m \geq 1, \\
& l_{j}^{2, \alpha}=\left(\frac{3}{2}\right)^{\alpha} \sum_{m=0}^{j} 3^{-m} l_{m}^{1, \alpha} l_{j-m}^{1, \alpha}, \\
& l_{k}^{3, \alpha}=\left(\frac{11}{6}\right)^{\alpha} \sum_{j=0}^{k} \sum_{m=0}^{j} \mu_{3}^{m} \bar{\mu}_{3}^{j-m} l_{m}^{1, \alpha} l_{j-m}^{1, \alpha} l_{k-j}^{1, \alpha}, \\
& l_{n}^{4, \alpha}=\left(\frac{25}{12}\right)^{\alpha} \sum_{k=0}^{n} \sum_{j=0}^{k} \sum_{m=0}^{j} \nu_{4}^{n-k} \mu_{4}^{m}{\overline{\mu_{4}}}^{j-m} l_{m}^{1, \alpha} l_{j-m}^{1, \alpha} l_{k-j}^{1, \alpha} l_{n-k}^{1, \alpha}, \\
& l_{q}^{5, \alpha}=\left(\frac{137}{60}\right)^{\alpha} \sum_{n=0}^{q} \sum_{k=0}^{n} \sum_{j=0}^{k} \sum_{m=0}^{j}{\overline{\nu_{5}^{q}}}^{q-n} \nu_{5}^{n-k} \mu_{5}^{m} \bar{\mu}^{j-m} l_{m}^{1, \alpha} l_{j-m}^{1, \alpha} l_{k-j}^{1, \alpha} l_{n-k}^{1, \alpha} l_{q-n}^{1, \alpha}
\end{aligned}
$$

with

$$
\begin{aligned}
& \mu_{3}=\frac{4}{7+\sqrt{39} i}, \overline{\mu_{3}}=\frac{4}{7-\sqrt{39} i}, i=\sqrt{-1}, \nu_{4}=\frac{3 a}{-b-\left(\sqrt[3]{Y_{1}}-\sqrt[3]{-Y_{2}}\right)}, \\
& \mu_{4}=\frac{3 a}{-b+\frac{1}{2}\left(\sqrt[3]{Y_{1}}-\sqrt[3]{-Y_{2}}\right)+\frac{\sqrt{3}}{2}\left(\sqrt[3]{Y_{1}}+\sqrt[3]{-Y_{2}}\right) i}, \overline{\mu_{4}}=\frac{3 a}{-b+\frac{1}{2}\left(\sqrt[3]{Y_{1}}-\sqrt[3]{-Y_{2}}\right)-\frac{\sqrt{3}}{2}\left(\sqrt[3]{Y_{1}}+\sqrt[3]{-Y_{2}}\right) i}, \\
& \nu_{5}=\frac{4}{-(b+M)+\sqrt{(b+M)^{2}-16\left(y+\frac{N}{M}\right)}}, \overline{\nu_{5}}=\frac{4}{-(b-M)+\sqrt{(b-M)^{2}-16\left(y-\frac{N}{M}\right)}}, \\
& \mu_{5}=\frac{4}{-(b+M)-\sqrt{(b+M)^{2}-16\left(y+\frac{N}{M}\right)}}, \overline{\mu_{5}}=\frac{4}{-(b-M)-\sqrt{(b-M)^{2}-16\left(y-\frac{N}{M}\right)}},
\end{aligned}
$$

where

$$
\begin{aligned}
& a=1, b=-\frac{21}{4}, c=\frac{137}{12}, d=-\frac{163}{12}, e=\frac{137}{12}, \widetilde{a}=1, \widetilde{b}=-\frac{137}{24}, \widetilde{c}=\frac{1231}{192}, \widetilde{d}=\frac{4259}{1536}, \\
& A=\widetilde{b}^{2}-3 \widetilde{a} \widetilde{c}, B=\widetilde{b} \widetilde{c}-9 \widetilde{a} \widetilde{d}, C=\widetilde{c}^{2}-3 \widetilde{b} \widetilde{d}, \Delta=B^{2}-4 A C<0, T=\frac{2 A \widetilde{b}-3 \widetilde{a} B}{2 A^{\frac{3}{2}}}, \theta=\arccos (T), \\
& y=\frac{-\widetilde{b}-2 A^{\frac{1}{2}} \cos \frac{\theta}{3}}{3 a}, M=\sqrt{8 y+b^{2}-4 c}, N=\sqrt{b y-d} .
\end{aligned}
$$

Acknowledgements. We thank Eli Barkai for the fruitful discussions and letting us know the urgency to solve the PDE with fractional substantial derivative in physical community. We thank the anonymous reviewer for suggesting us to add Remark 4.14 and Algorithms 1-2.

\section{REFERENCES}

[1] E. Barkai and R.J. Silbey, Fractional Kramers equation. J. Phys. Chem. B 104 (2000) 3866-3874.

[2] S. Carmi and E. Barkai, Fractional Feynman-Kac equation for weak ergodicity breaking. Phys. Rev. E 84 (2011) 061104.

[3] S. Carmi, L. Turgeman and E. Barkai, On distributions of functionals of anomalous diffusion paths. J. Stat. Phys. 141 (2010) 1071-1092. 
[4] M.H. Chen and W.H. Deng, Fourth order difference approximations for space Riemann-Liouville derivatives based on weighted and shifted Lubich difference operators. Commun. Comput. Phys. 16 (2014) 516-540.

[5] M.H. Chen and W.H. Deng, Fourth order accurate scheme for the space fractional diffusion equations. SIAM J. Numer. Anal. 52 (2014) 1418-1438.

[6] W.H. Deng, M.H. Chen and E. Barkai, Numerical algorithms for the forward and backward fractional Feynman-Kac equations. J. Sci. Comput. (2014). DOI: 10.1007/s10915-014-9873-6.

[7] G.M. Fikhtengoltz, Course of Differential and Integral Calculus, vol. 2. Nauka, Moscow (1969).

[8] R. Friedrich, F. Jenko, A. Baule and S. Eule, Anomalous diffusion of inertial, weakly damped particles. Phys. Rev. Lett. 96 (2006) 230601.

[9] R. Gorenflo and F. Mainardi, Fractional calculus: intergtal and differential equations of fractional order. Fractals and Fractional Calculus in Continuum Mechanics, edited by A. Carpinteri and F. Mainardi. Springer Verlag, Wien and New York (1997).

[10] P. Henrici, Discrete Variable Methods in Ordinary Differential Equations. John Wiley, New York (1962).

[11] Ch. Lubich, Discretized fractional calculus. SIAM J. Math. Anal. 17 (1986) 704-719.

[12] R. Metzler and J. Klafter, The random walk's guide to anomalous diffusion: A fractional dynamics approach. Phys. Rep. 339 (2000) $1-77$.

[13] R. Metzler and T.F. Nonnenmacher, Fractional diffusion, waiting-time distributions, and Cattaneo-type equations. Phys. Rev. E 57 (1998) 6409.

[14] R. Metzler and I.M. Sokolov, Superdiffusive Klein-Kramers equation: Normal and anomalous time evolution and Lévy walk moments. Europhys. Lett. 58 (2002) 482-488.

[15] K.S. Miller and B. Ross, An Introduction to the Fractional Calculus and Fractional Differential Equations. Wiley-Interscience Publication, USA (1993).

[16] K.B. Oldham and J. Spanier, The Fractional Calculus. Academic Press, New York (1974).

[17] I. Podlubny, Fractional Differential Equations. Academic Press, New York (1999).

[18] I.M. Sokolov and R. Metzler, Towards deterministic equations for Lévy walks: The fractional material derivative. Phys. Rev. E 67 (2003) 010101(R).

[19] W.Y. Tian, H. Zhou and W.H. Deng, A class of second order difference approximations for solving space fractional diffusion equations. To appear in Math. Comput. (2015). Doi:10.1090/S0025-5718-2015-02917-2.

[20] L. Turgeman, S. Carmi and E. Barkai, Fractional Feynman-Kac Equation for Non-Brownian Functionals. Phys. Rev. Lett. 103 (2009) 190201. 